\title{
Association of Serum Fatty Acids at Admission with the Age of Onset of Acute Ischemic Stroke
}

\author{
Takahisa Mori * (D), Kazuhiro Yoshioka, Yuhei Tanno and Shigen Kasakura \\ Department of Stroke Treatment, Shonan Kamakura General Hospital, Okamoto 1370-1, Kamakura City, Japan; \\ y.kazuhiro12@icloud.com (K.Y.); yxip01@icloud.com (Y.T.); kasakura@med.kitasato-u.ac.jp (S.K.) \\ * Correspondence: morit-koc@umin.net; Tel.: +81-467-461-717
}

Received: 11 July 2020; Accepted: 11 August 2020; Published: 12 August 2020

check for updates

\begin{abstract}
Dietary triglycerides influence fatty acid (FA) serum concentrations and weight percentages (wt \%), which may be associated with the age of onset of acute ischemic stroke (AIS). We investigated the correlations between serum FA levels and proportions at admission and the age of onset of AIS. We evaluated patients with AIS admitted between 2016 and 2019 within $24 \mathrm{~h}$ of AIS onset and calculated the correlation coefficients between their ages, serum FA concentrations, and FA wt \% values. Multiple linear regression analysis was performed to identify independent FAs indicating AIS age of onset. Furthermore, we estimated the threshold values of independent FAs for age of onset $<60$ years using receiver operating characteristic curves by logistic regression. A total of 525 patients (median age: 75 years) met the inclusion criteria. The concentration of dihomo-gamma-linolenic acid (DGLA) and wt \% of docosahexaenoic acid (DHA) were significant independent variables for age of onset of AIS, and receiver operating characteristic curves for age of onset $<60$ years showed thresholds of $\geq 117.7 \mu \mathrm{mol} / \mathrm{L}$ for DGLA and $\leq 3.7 \%$ for DHA. An increased DGLA concentration and decreased DHA wt $\%$ were significantly associated with onset of AIS at a younger age.
\end{abstract}

Keywords: serum fatty acids; admission; acute ischemic stroke; age of onset; dihomo-gamma-linolenic acid; docosahexaenoic acid

\section{Introduction}

Elevated serum triglycerides (TGs), which consist of glycerol and three fatty acids (FAs), are a risk factor for vascular disease [1]. The incidence of ischemic vascular disease increases with increasing age. Dietary TGs, which are derived from meat, fish, and vegetables, influence serum TG and FA levels [2]. Higher consumption of fish and n-3 polyunsaturated fatty acids (n-3 PUFAs) is associated with a reduced risk of thrombotic infarction [3] and coronary artery disease (CAD) [4] in healthy middle-aged subjects. Previous studies have reported the neuroprotective effects of docosahexaenoic acid (DHA) [5-7]. The relationship between serum FA levels under fasting conditions and stroke has been reported [8-10]. However, few studies have reported on serum FA levels at the onset of ischemic events [11,12], which are typically examined under non-fasting conditions and associated with the dietary intake of TGs. Higher intake of seafood may be associated with higher n-3 PUFA levels, which is likely to reduce the risk of ischemic events.

A low serum n-3 PUFA/n-6 PUFA ratio may be associated with neurological deterioration in patients with acute ischemic stroke (AIS) [13], and low serum n-3 PUFA levels may also be associated with the onset of AIS.

The weight percentages (wt \%) of individual FAs, with respect to total serum FAs, have been examined in healthy humans. With increasing age, the wt $\%$ of $n-6$ PUFAs and n-3 PUFAs decrease and increase, respectively [14]. CAD results in increased serum concentrations of palmitic acid (PA), stearic acid (StA), oleic acid (OlA), linoleic acid (LiA), and arachidonic acid (AA) and a lower serum 
concentration of eicosapentaenoic acid (EPA) compared to subjects without CAD. Additionally, the wt $\%$ values of EPA and DHA are lower in subjects with CAD than in healthy controls [15]. Interestingly, in patients who experienced lacunar or atherosclerotic stroke between the ages of 50-74 years, the serum concentrations of saturated fatty acids (SFAs), n-9 monounsaturated fatty acid (n-9 MUFA), and n-6 PUFAs are elevated [11,12]. This suggests that the serum concentrations and wt $\%$ of FAs are related to the age of acute ischemic disease onset. Dietary DHA has beneficial effects on neurodevelopment in mice [16]. DHA and AA are rich in phospholipids and are found in the central nervous system [17]. In this study, we investigated the associations between the serum concentrations and proportions of FAs in patients admitted for AIS and age of AIS onset.

\section{Materials and Methods}

We conducted a cross-sectional study of patients with AIS who were admitted to our institution between August 2016 and July 2019 within 24 h of AIS onset and underwent evaluation of blood lipids and FAs at admission. We excluded patients with a pre-hospital modified Rankin scale score $\geq 3$ or body mass index $<18.5$, who were defined as having a severe disability, or who were underweight, respectively, according to World Health Organization guidelines. This was performed to exclude patients with possible malnutrition.

\subsection{Measurement of Serum Lipids and FAs}

Serum total cholesterol (T-CHO), TGs, and high-density lipoprotein-cholesterol (HDL-C) were measured enzymatically using reagents manufactured by Denka Seiken (Denka Co., Ltd., Chuo, Tokyo, Japan) on a BioMajesty 6050 High Throughput Clinical Chemistry Analyzer (JEOL Ltd., Akishima, Tokyo, Japan). Low-density lipoprotein-cholesterol (LDL-C) was calculated using the Friedewald formula as follows: LDL-C $=(\mathrm{T}-\mathrm{CHO}-\mathrm{HDL}-\mathrm{C}-\mathrm{TG}) / 5$. We examined the SFAs lauric acid (LaA), myristic acid (MyA), PA, and StA; the n-9 MUFA OlA; the n-6 PUFAs LiA, dihomo-gamma-linolenic acid (DGLA), and AA; and the n-3 PUFAs alpha-linolenic acid (AlA), EPA, and DHA. We measured the serum concentrations and wt \% of each FA at admission. The FAs in $1 \mathrm{~mL}$ of serum were measured. FAs were extracted according to a general technique using tricosanoic acid (Nu-Chek Prep, Inc., Elysian, MN, USA) as an internal standard. Lipid extracts were hydrolyzed, extracted with chloroform, and dried under nitrogen gas. After adding 30\% potassium methoxide methanol solution to the residual sample, the sample was incubated at $100{ }^{\circ} \mathrm{C}$ for $5 \mathrm{~min}$ and cooled. Samples were extracted with hexane and analyzed on a GC-2010 Plus Capillary Gas Chromatograph (Shimadzu, Kyoto, Japan) equipped with a flame ionization detector, using a BPX70 column. The operating conditions were as follows: $50{ }^{\circ} \mathrm{C}$ for $0.5 \mathrm{~min}$, temperature increase to $260{ }^{\circ} \mathrm{C}$ over $25 \mathrm{~min}$, and holding at this temperature for $5 \mathrm{~min}$. The injector and detector temperatures were $240{ }^{\circ} \mathrm{C}$ and $280^{\circ} \mathrm{C}$, respectively, and helium was used as the carrier gas at $1.09 \mathrm{~mL} / \mathrm{min}$. Component identification was performed by comparing the retention times of the samples with those of standards. We evaluated the concentrations of serum lipids and FAs and wt \% of serum FAs using internal standard ratios.

\subsection{Ethical Approval and Consent to Participate}

All procedures were performed in accordance with the ethical standards of the institution and the 1964 Helsinki Declaration. The Tokusyukai Group Ethical Committee approved our retrospective study (TGE01486-024).

\subsection{Consent to Participate}

Written informed consent for participation and publication was not required. The study was based on an opt-out model of enrolment, which was permitted by the ethical committee. 


\subsection{Statistical Analysis}

We expressed non-normally distributed continuous variables as medians and interquartile ranges. We used a multiple comparison test to compare all possible FA pairs and Spearman rank correlation coefficient $\left(r_{\mathrm{s}}\right)$ to measure the strength of the relationships between non-normally distributed variables. We defined $0 \leq\left|r_{\mathrm{s}}\right|<0.1$ as no correlation, $0.1 \leq\left|r_{\mathrm{s}}\right|<0.4$ as a weak correlation, $0.4 \leq\left|r_{\mathrm{s}}\right|<0.6$ as a moderate correlation, and $0.6 \leq\left|r_{\mathrm{s}}\right|$ as a strong correlation. Multicollinearity was defined as a strong correlation between variables. After excluding FAs with multicollinearity, we performed multiple linear regression analysis to identify independent FAs affecting the AIS age of onset. Multiple logistic regression analysis was also used to identify independent FAs correlated with ages of onset of $<60$ and $<80$ years. We estimated the threshold values of FAs for onset at age $<60$ or $<80$ years using area under the curve (AUC) values derived from the receiver operating characteristic (ROC) curves of the logistic regression model. A probability $p<0.05$ was considered as statistically significant. We used the JMP software (version 15.1; SAS Institute, Cary, NC, USA) for all statistical analyses.

\section{Results}

A total of 880 patients with AIS were admitted to our stroke center during the study period, and 525 patients met our inclusion criteria. Patient characteristics are summarized in Table 1 AIS occurred in 61 patients $(11.6 \%)$ aged $<60$ years and in 341 patients $(65.0 \%)$ aged $<80$ years. Age was negatively correlated with most FA concentrations and FA wt \% values, except for n-3 PUFAs (Table 2).

Table 1. Patient characteristics.

\begin{tabular}{|c|c|}
\hline & $n=525$ \\
\hline Age (MD, IQR) (min, max) years & 75 (68-81) (min: 39, max: 99) \\
\hline Male sex & $337(64.2 \%)$ \\
\hline Height (MD, IQR) cm & $163(155-168)$ \\
\hline Body weight (MD, IQR) kg & $60(53-68.5)$ \\
\hline Body mass index (MD, IQR) kg/m² & $23.1(21.0-25.4)$ \\
\hline \multicolumn{2}{|l|}{ Glucose and lipids } \\
\hline Blood sugar (MD, IQR) mmol/L & $6.83(5.94-8.49)$ \\
\hline Hemoglobin A1c (MD, IQR)\% (NGSP) & $5.9(5.6-6.4)$ \\
\hline Total cholesterol (MD, IQR) $\mathrm{mmol} / \mathrm{L}$ & $5.09(4.42-5.84)$ \\
\hline High-density lipoprotein cholesterol (MD, IQR) mmol/L & $1.43(1.19-1.74)$ \\
\hline Triglycerides (MD, IQR) $\mathrm{mmol} / \mathrm{L}$ & $1.19(0.81-1.80)$ \\
\hline \multicolumn{2}{|l|}{ Saturated fatty acids } \\
\hline Lauric acid (LaA) (MD, IQR) $\mu \mathrm{mol} / \mathrm{L}$ & $4.49(2.99-8.48)$ \\
\hline Myristic acid (MyA) (MD, IQR) $\mu \mathrm{mol} / \mathrm{L}$ & $70.08(52.12-97.67)$ \\
\hline Palmitic acid (PA) (MD, IQR) $\mu \mathrm{mol} / \mathrm{L}$ & $2,472(2,109-2,903)$ \\
\hline Stearic acid (StA) (MD, IQR) $\mu \mathrm{mol} / \mathrm{L}$ & $638.5(544.9-747.3)$ \\
\hline $\mathrm{LaA}(\mathrm{MD}, \mathrm{IQR}) \%$ & $0(0-0.1)$ \\
\hline MyA (MD, IQR)\% & $0.6(0.5-0.8)$ \\
\hline PA (MD, IQR)\% & $23.6(22.6-24.5)$ \\
\hline StA (MD, IQR)\% & $6.7(6.2-7.2)$ \\
\hline \multicolumn{2}{|l|}{ n-9 MUFA } \\
\hline Oleic acid (OlA) (MD, IQR) $\mu \mathrm{mol} / \mathrm{L}$ & $2,008(1,656-2,527)$ \\
\hline OlA (MD, IQR)\% & $21.1(19.4-23.2)$ \\
\hline \multicolumn{2}{|l|}{ n-6 PUFAs } \\
\hline Linoleic acid (LiA) (MD, IQR) $\mu \mathrm{mol} / \mathrm{L}$ & $2,515(2,150-2,942)$ \\
\hline Dihomo-gamma-linolenic acid (DGLA) (MD, IQR) $\mu \mathrm{mol} / \mathrm{L}$ & $89.98(69.76-115.57)$ \\
\hline Arachidonic acid (AA) (MD, IQR) $\mu \mathrm{mol} / \mathrm{L}$ & $517.9(439.0-604.5)$ \\
\hline $\mathrm{LiA}(\mathrm{MD}, \mathrm{IQR}) \%$ & $26.1(23.4-28.4)$ \\
\hline DGLA (MD, IQR)\% & $1(0.8-1.2)$ \\
\hline AA (MD, IQR $\%$ & $5.9(4.9-6.7)$ \\
\hline
\end{tabular}


Table 1. Cont.

\begin{tabular}{cc}
\hline & $n=525$ \\
\hline n-3 PUFAs & \\
Alpha-linolenic acid (AlA) (MD, IQR) $\mu \mathrm{mol} / \mathrm{L}$ & $65.34(49.00-87.42)$ \\
Eicosapentaenoic acid (EPA) (MD, IQR) $\mu \mathrm{mol} / \mathrm{L}$ & $206.2(142.2-302.70)$ \\
Docosahexaenoic acid (DHA) (MD, IQR) $\mu \mathrm{mol} / \mathrm{L}$ & $376.66(303.54-469.38)$ \\
AlA (MD, IQR) $\%$ & $0.7(0.6-0.8)$ \\
EPA (MD, IQR)\% & $2.2(1.6-3.4)$ \\
DHA (MD, IQR) $\%$ & $4.6(3.7-5.5)$ \\
EPA/AA ratio (MD, IQR) & $0.39(0.265-0.575)$ \\
n-6/n-3 ratio (MD, IQR) & $4.16(3.2-5.315)$ \\
\hline
\end{tabular}

Min: minimum, max: maximum, MD: median, IQR: interquartile range; NGSP: National Glycohemoglobin Standardization Program; wt \%: weight percentage of total fatty acids; n-3 PUFA: n-3 polyunsaturated fatty acid; n-6 PUFA: n-6 polyunsaturated fatty acid; n-9 MUFA: n-9 monounsaturated fatty acid.

Table 2. Spearman correlation coefficients.

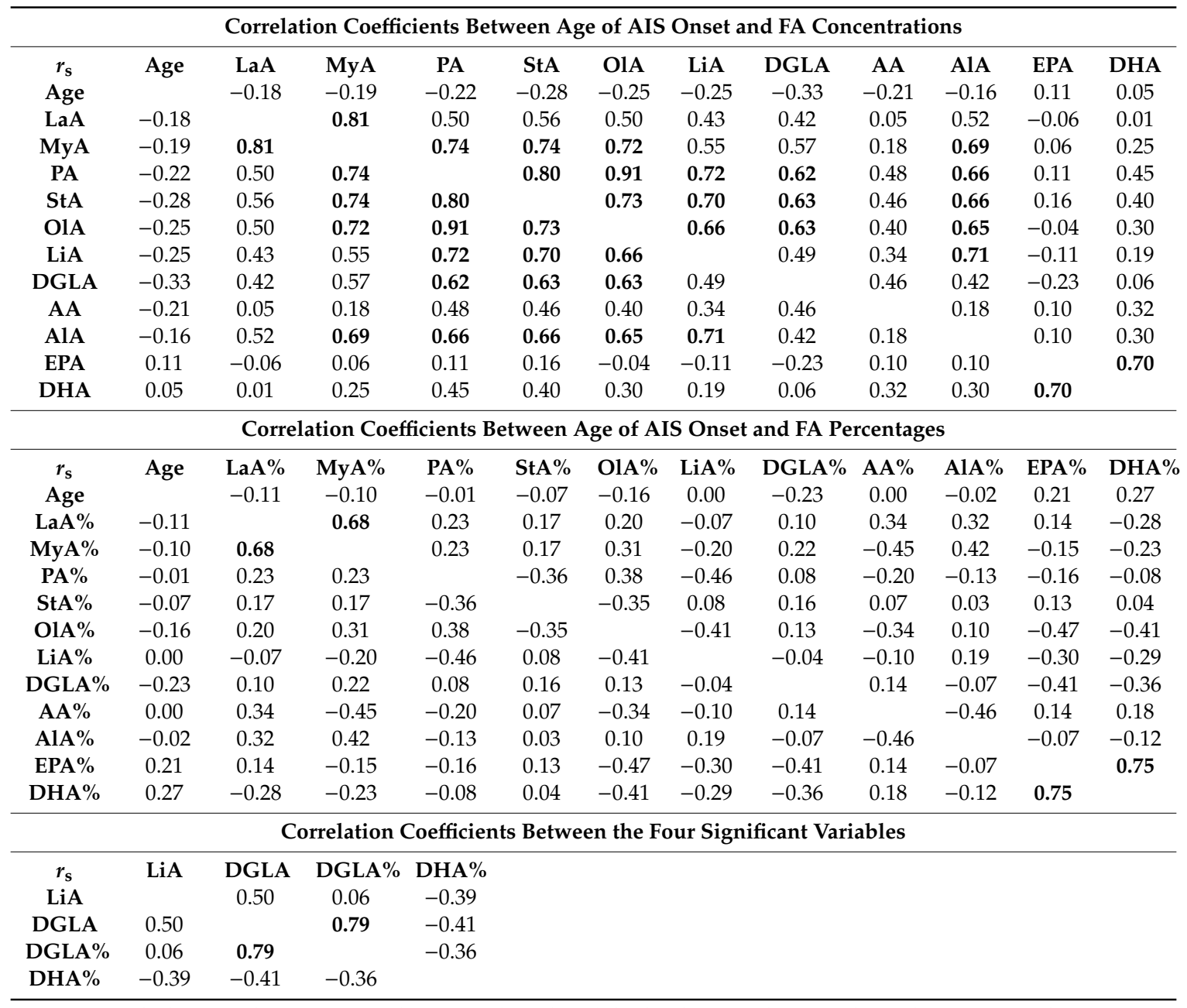

AA: arachidonic acid; AIS: acute ischemic stroke; AlA: alpha-linolenic acid; DHA: docosahexaenoic acid; DGLA: dihomo-gamma-linolenic acid; EPA: eicosapentaenoic acid; LaA: lauric acid; LiA: linoleic acid; MyA: myristic acid; OlA: oleic acid; PA: palmitic acid; StA: stearic acid.

After excluding FA concentrations with multicollinearity and those without a correlation with age, we performed multiple linear regression analysis using the MyA, LiA, DGLA, AA, and EPA concentrations as explanatory variables. The results revealed that LiA and DGLA were independent variables when the age at AIS onset was an objective variable (Table 3 ). We also performed multiple linear regression analysis using the LaA, OlA, DGLA, and DHA wt \% values as explanatory variables 
and found that DGLA wt \% and DHA wt \% were independent variables when the age ofAIS onset was an objective variable (Table 3).

Table 3. Multiple linear regression analysis of the age of AIS onset.

\begin{tabular}{|c|c|c|c|}
\hline Between FA concentrations & & & \\
\hline Variables & $t$-value & $p$ & adjusted $R^{2}$ \\
\hline Myristic acid & 1.43 & ns & 0.142 \\
\hline Linoleic acid (LiA) & -2.63 & $<0.01$ & \\
\hline Dihomo-gamma-linolenic acid (DGLA) & -4.68 & $<0.0001$ & \\
\hline Arachidonic acid & -1.63 & ns & \\
\hline Eicosapentaenoic acid & 0.55 & ns & \\
\hline \multicolumn{4}{|l|}{ Between FA percentages } \\
\hline Variables & $t$-value & $p$ & adjusted $R^{2}$ \\
\hline Lauric acid wt \% & 0.87 & ns & 0.119 \\
\hline Oleic acid wt \% & -1.42 & ns & \\
\hline DGLA wt $\%$ & -3.66 & $<0.001$ & \\
\hline Docosahexaenoic acid (DHA) wt \% & 4.75 & $<0.0001$ & \\
\hline \multicolumn{4}{|l|}{ Between LiA, DGLA, and DHA wt \% } \\
\hline Variables & $t$-value & $p$ & adjusted $R^{2}$ \\
\hline $\mathrm{LiA}$ & -1.61 & ns & 0.160 \\
\hline DGLA & -4.72 & $<0.0001$ & \\
\hline DHA\% & 3.96 & $<0.0001$ & \\
\hline
\end{tabular}

AIS: acute ischemic stroke; ns, not significant.

Next, after excluding the DGLA wt \% because of multicollinearity (Table 2), we performed multiple linear regression analysis using the LiA and DGLA concentrations and DHA wt \%, which demonstrated that the DGLA concentration and DHA wt \% were independent variables when age at AIS onset was an objective variable (Table 3). Multiple logistic regression analysis showed that DGLA concentration and DHA wt $\%$ were significant variables when the objective variable was age of onset $<60$ years (AUC: 0.810 ) and $<80$ years (AUC: 0.648). The ROC curves estimated threshold values for the DGLA concentration and DHA wt \% for onset at age $<60$ and $<80$ years are shown in Table 4 .

Table 4. Threshold values for age of AIS onset using receiver operating curves from logistic regression analysis.

\begin{tabular}{|c|c|c|c|c|c|c|c|c|c|}
\hline & $N$ & $\begin{array}{c}\text { Sens } \\
(\%)\end{array}$ & $\begin{array}{c}\text { Spec } \\
(\%)\end{array}$ & $\begin{array}{l}\text { PPV } \\
(\%)\end{array}$ & Odds ratio & $p$ & AUC & AICc & BIC \\
\hline Age $<60$ years as an $\mathrm{OV}$ & & & & & & & & & \\
\hline DGLA ( $\geq 117.7$ vs. $<117.7) \mu \mathrm{mol} / \mathrm{L}$ & 525 & 55.7 & 81.3 & 28.1 & $1.07(1.05-1.09)$ & $<0.0001$ & 0.727 & 339 & 348 \\
\hline $\begin{array}{c}\text { DHA wt } \%(\leq 3.7 \text { vs. }>3.7) \% \\
\text { Age }<80 \text { years as an OV }\end{array}$ & 525 & 68.9 & 80.2 & 31.3 & $0.36(0.27-0.48)$ & $<0.0001$ & 0.792 & 315 & 323 \\
\hline DGLA ( $\geq 76.3$ vs. $<76.3) \mu \mathrm{mol} / \mathrm{L}$ & 525 & 76 & 48.9 & 73.4 & $1.05(1.03-1.07)$ & $<0.0001$ & 0.535 & 657 & 666 \\
\hline DHA wt $\%(\leq 3.8$ vs. $>3.8) \%$ & 525 & 32.8 & 82.1 & 77.2 & $0.75(0.65-0.87)$ & $<0.0001$ & 0.600 & 668 & 677 \\
\hline
\end{tabular}

AIS: acute ischemic stroke; AICc: corrected Akaike information criterion; AUC: area under the curve, BIC: Bayesian information criterion; DGLA: dihomo-gamma-linolenic acid; DHA: docosahexaenoic acid; OV: objective variable; PPV: positive predictive value; Sens: sensitivity, Spec: specificity.

\section{Discussion}

Our results demonstrate that in patients with AIS, the DGLA concentration and DHA wt $\%$ are significant independent variables for the age of AIS onset and that increased DGLA concentrations and decreased DHA proportions are significantly associated with young-onset AIS.

Except for DHA, FA concentrations were positively correlated with the age of AIS onset; the levels of LiA and DGLA (n-6 PUFAs) were strongly correlated with the levels of PA and StA (SFAs), as well as OlA (an n-9 MUFA). There were strong correlations between various SFA concentrations. The OlA 
concentration was strongly correlated with the levels of SFAs, LiA, and DGLA (n-6 PUFAs), and AlA (n-3 PUFA). The EPA concentration was strongly correlated with that of DHA (Table 2). These strong mutual correlations were related to the ingestion of certain foods, including large amounts of various FAs, as recent dietary quantities influenced serum concentrations at admission. The wt \% values of FAs, which reflected the dietary FA composition, may be influenced not by the quantity of ingested FAs but rather by their quality. The wt \% values of five FAs (LaA, OlA, DGLA, EPA, and DHA) were correlated with the age of AIS onset, and EPA wt \% was strongly correlated with the DHA wt \%. FA wt \% values displayed quite different trends from FA concentrations.

A previous study reported that elevated serum PA and OlA levels are associated with an increased frequency of incidental lacunar stroke and that elevated serum DHA and AA levels are associated with a decreased incidence of ischemic stroke [9]. However, the results were obtained in a case-controlled study, in which blood was collected under fasting conditions from all participants, and serum FA levels were not assessed at admission at AIS onset. In our series, the concentrations of PA, OlA, and AA at admission showed weak negative correlations with the age of AIS onset, although they were not independent variables.

In a study of healthy Canadians in their $20 \mathrm{~s}$, the median concentrations of LiA, DGLA, and DHA were 2208.2, 68.2, and $82.0 \mu \mathrm{mol} / \mathrm{L}$, respectively [18], which are lower than those detected in our study. Particularly, the median DHA concentration in our study was $376.7 \mu \mathrm{mol} / \mathrm{L}$, which is much higher than that in healthy young people. In a study of patients with CAD in the United States with an average age of 47 years, the wt \% of LiA, AA, EPA, and DHA were $58.6 \%, 9.4 \%, 0.9 \%$, and $0.5 \%$, respectively [15]. The proportions of LiA and AA were higher than those in the US patients compared to in our patients; however, the proportions of EPA, and DHA were lower. The median age in our study was 75 years, and $35 \%$ of patients were aged 80 years or older. The DHA wt \% in our patients was much higher than that in the US patients ( $4.6 \%$ vs. $0.5 \%)$.

It has been suggested that n-3 PUFAs can reduce the incidence of CAD and stroke, as well as mortality associated with cardiovascular disease [19-23]. Administration of purified EPA reduced recurrent stroke [21]. In contrast, SFAs may increase the risk of these conditions [24]. In our patients, the concentrations and proportions of SFAs and EPA were not independent variables for the age of AIS onset; however, DHA wt \% was an independent variable, and elevated DHA wt \% values were associated with late-onset AIS.

Dietary intake of TGs, which are found in meat, fish, vegetables, and their oils, influences serum FA concentrations and proportions. Therefore, it is essential to assess the types and quantities of dietary TG sources consumed during the days prior to AIS onset. Dietary intake assessment and developing dietary regimens that decrease the DGLA concentration and increase the DHA proportion may prevent AIS or delay its onset. A prospective randomized control study is required to identify the appropriate intake of FAs to achieve these goals.

Author Contributions: Conceptualization, T.M.; Methodology, T.M.; Validation, T.M.; Formal Analysis, T.M.; Investigation, T.M., K.Y., Y.T., and S.K.; Resources, T.M., K.Y., Y.T., and S.K.; Data Curation, T.M., K.Y., Y.T., and S.K.; Writing-Original Draft Preparation, T.M.; Writing-Review \&Editing, T.M.; Visualization, T.M.; Supervision, T.M.; Final approval of the submitted version, T.M., K.Y., Y.T., and S.K. All authors have read and agreed to the published version of the manuscript.

Funding: This research received no external funding.

Acknowledgments: The authors wish to thank Nozomi Chiba, Bachelor of Arts (BA), for secretarial support. The authors received no specific funding for this work.

Conflicts of Interest: The authors have no conflicts of interest to disclose. 


\section{References}

1. Talayero, B.G.; Sacks, F.M. The role of triglycerides in atherosclerosis. Curr. Cardiol. Rep. 2011, 13, 544-552. [CrossRef]

2. von Lossonczy, T.O.; Ruiter, A.; Bronsgeest-Schoute, H.C.; van Gent, C.M.; Hermus, R.J. The effect of a fish diet on serum lipids in healthy human subjects. Am. J. Clin. Nutr. 1978, 31, 1340-1346. [CrossRef] [PubMed]

3. Iso, H.; Rexrode, K.M.; Stampfer, M.J.; Manson, J.E.; Colditz, G.A.; Speizer, F.E.; Hennekens, C.H.; Willett, W.C. Intake of fish and omega-3 fatty acids and risk of stroke in women. JAMA 2001, 285, 304-312. [CrossRef] [PubMed]

4. Iso, H.; Kobayashi, M.; Ishihara, J.; Sasaki, S.; Okada, K.; Kita, Y.; Kokubo, Y.; Tsugane, S.; Group, J.S. Intake of fish and $\mathrm{n} 3$ fatty acids and risk of coronary heart disease among Japanese: The Japan Public Health Center-Based (JPHC) Study Cohort I. Circulation 2006, 113, 195-202. [CrossRef] [PubMed]

5. Yang, B.; Li, R.; Michael Greenlief, C.; Fritsche, K.L.; Gu, Z.; Cui, J.; Lee, J.C.; Beversdorf, D.Q.; Sun, G.Y. Unveiling anti-oxidative and anti-inflammatory effects of docosahexaenoic acid and its lipid peroxidation product on lipopolysaccharide-stimulated BV-2 microglial cells. J. Neuroinflamm. 2018, 15, 202. [CrossRef]

6. Geng, X.; Yang, B.; Li, R.; Teng, T.; Ladu, M.J.; Sun, G.Y.; Greenlief, C.M.; Lee, J.C. Effects of Docosahexaenoic Acid and Its Peroxidation Product on Amyloid- $\beta$ Peptide-Stimulated Microglia. Mol. Neurobiol. 2020, 57, 1085-1098. [CrossRef]

7. Yang, B.; Li, R.; Liu, P.N.; Geng, X.; Mooney, B.P.; Chen, C.; Cheng, J.; Fritsche, K.L.; Beversdorf, D.Q.; Lee, J.C.; et al. Quantitative Proteomics Reveals Docosahexaenoic Acid-Mediated Neuroprotective Effects in Lipopolysaccharide-Stimulated Microglial Cells. J. Proteome Res. 2020, 19, 2236-2246. [CrossRef]

8. Simon, J.A.; Fong, J.; Bernert, J.T., Jr.; Browner, W.S. Serum fatty acids and the risk of stroke. Stroke 1995, 26, 778-782. [CrossRef]

9. Yaemsiri, S.; Sen, S.; Tinker, L.F.; Robinson, W.R.; Evans, R.W.; Rosamond, W.; Wasserthiel-Smoller, S.; $\mathrm{He}, \mathrm{K}$. Serum fatty acids and incidence of ischemic stroke among postmenopausal women. Stroke 2013, 44, 2710-2717. [CrossRef]

10. Yamagishi, K.; Folsom, A.R.; Steffen, L.M.; Investigators, A.S. Plasma fatty acid composition and incident ischemic stroke in middle-aged adults: The atherosclerosis risk in communities (ARIC) study. Cerebrovasc. Dis. 2013, 36, 38-46. [CrossRef]

11. Mori, T.; Tanno, Y.; Kasakura, S.; Yoshioka, K.; Nakai, N. Serum fatty acids and ischemic stroke subtypes in middle- and late-onset acute stroke patients. Clin. Nutr. Exp. 2018, 22, 19-29. [CrossRef]

12. Mori, T.; Yoshioka, K. Features of serum fatty acids in acute ischaemic stroke patients aged 50 years or older. BMC Cardiovasc. Disord. 2020, 20, 122. [CrossRef]

13. Suda, S.; Katsumata, T.; Okubo, S.; Kanamaru, T.; Suzuki, K.; Watanabe, Y.; Katsura, K.; Katayama, Y. Low serum n-3 polyunsaturated fatty acid/n-6 polyunsaturated fatty acid ratio predicts neurological deterioration in Japanese patients with acute ischemic stroke. Cerebrovasc. Dis. 2013, 36, 388-393. [CrossRef] [PubMed]

14. Takita, T.; Nakamura, K.; Kimira, M.; Yamada, N.; Kobayashi, Y.; Innami, S. Serum fatty acid compositions and lipid concentrations and their correlations. J. Clin. Biochem. Nutr. 1996, 20, 149-159. [CrossRef]

15. Schwertner, H.; Mosser, E. Comparison of lipid fatty acids on a concentration basis vs. weight percentage basis in patients with and without coronary artery disease or diabetes. Clin. Chem. 1993, 39, 659-663. [CrossRef] [PubMed]

16. Yang, B.; Li, R.; Woo, T.; Browning, J.D., Jr.; Song, H.; Gu, Z.; Cui, J.; Lee, J.C.; Fritsche, K.L.; Beversdorf, D.Q.; et al. Maternal dietary docosahexaenoic acid alters lipid peroxidation products and (n-3)/(n-6) fatty acid balance in offspring mice. Metabolites 2019, 9, 40. [CrossRef] [PubMed]

17. Yang, B.; Fritsche, K.L.; Beversdorf, D.Q.; Gu, Z.; Lee, J.C.; Folk, W.R.; Greenlief, C.M.; Sun, G.Y. Yin-Yang mechanisms regulating lipid peroxidation of docosahexaenoic acid and arachidonic acid in the central nervous system. Front. Neurol. 2019, 10, 642. [CrossRef]

18. Abdelmagid, S.A.; Clarke, S.E.; Nielsen, D.E.; Badawi, A.; El-Sohemy, A.; Mutch, D.M.; Ma, D.W. Comprehensive profiling of plasma fatty acid concentrations in young healthy Canadian adults. PLoS ONE 2015, 10, e0116195. [CrossRef]

19. Dyerberg, J.; Bang, H.O. A hypothesis on the development of acute myocardial infarction in Greenlanders. J. Clin. Lab. Invest. Suppl. 1982, 161, 7-13. [CrossRef] 
20. Kromhout, D.; Bosschieter, E.B.; de Lezenne Coulander, C. The inverse relation between fish consumption and 20-year mortality from coronary heart disease. N. Engl. J. Med. 1985, 312, 1205-1209. [CrossRef]

21. Tanaka, K.; Ishikawa, Y.; Yokoyama, M.; Origasa, H.; Matsuzaki, M.; Saito, Y.; Matsuzawa, Y.; Sasaki, J.; Oikawa, S.; Hishida, H.; et al. Reduction in the recurrence of stroke by eicosapentaenoic acid for hypercholesterolemic patients: Subanalysis of the JELIS trial. Stroke 2008, 39, 2052-2058. [CrossRef] [PubMed]

22. Miyagawa, N.; Miura, K.; Okuda, N.; Kadowaki, T.; Takashima, N.; Nagasawa, S.Y.; Nakamura, Y.; Matsumura, Y.; Hozawa, A.; Fujiyoshi, A.; et al. Long-chain n-3 polyunsaturated fatty acids intake and cardiovascular disease mortality risk in Japanese: A 24-year follow-up of NIPPON DATA80. Atherosclerosis 2014, 232, 384-389. [CrossRef] [PubMed]

23. Morise, A.; Sérougne, C.; Gripois, D.; Blouquit, M.-F.; Lutton, C.; Hermier, D. Effects of dietary alpha linolenic acid on cholesterol metabolism in male and female hamsters of the LPN strain. J. Nutr. Biochem. 2004, 15, 51-61. [CrossRef] [PubMed]

24. Sacks, F.M.; Lichtenstein, A.H.; Wu, J.H.Y.; Appel, L.J.; Creager, M.A.; Kris-Etherton, P.M.; Miller, M.; Rimm, E.B.; Rudel, L.L.; Robinson, J.G.; et al. Dietary fats and cardiovascular disease: A presidential advisory from the American Heart Association. Circulation 2017, 136, e1-e23. [CrossRef]

(C) 2020 by the authors. Licensee MDPI, Basel, Switzerland. This article is an open access article distributed under the terms and conditions of the Creative Commons Attribution (CC BY) license (http://creativecommons.org/licenses/by/4.0/). 